\title{
AIRBORNE MEASUREMENT OF ATMOSPHERIC TURBULENCE
}

by

\author{
William C. Rose \\ Rose Engineering and Research, Inc. \\ Incline Village, Nevada 89450 \\ and \\ Leonard J. Otten, III, Major USAF \\ AFWL/LR- 2 \\ Kirtland AFB, NM 87117 \\ Paper presented at \\ USAF / NASA Aero-Optics Symposium \\ 14-15 August 1979 \\ NASA Ames Research Center \\ Moffett Field, California 94035
}




\begin{abstract}
A system is described that is capable of making measurements of fluctuating atmospheric density and spatial scales required in assessing the quality of coherent radiation propogation. The airborne platform for the system is an NC-135 Air Force research aircraft. This system is completely operational and has flown successfully for approximately 60 hours to date. Scales from hundreds of meters to millimeters have been measured from near ground level to $12 \mathrm{~km}$. A heated and unheated fine wire sensor are used to produce signals from the atmospheric turbulence as the aircraft flies through the turbulence. These signals are analyzed using techniques developed for use in high-speed wind tunnels. With these data interpretation techniques available, the aircraft Mach number may be arbitrarily high -- even supersonic flight presents no problem. With such high velocities possible, extensive regions of the atmosphere may be traversed in a relatively short time. This report will describe the special sensors, aircraft installation, data reduction procedures, and other special requirements necessary to obtain meaningful atmospheric turbulence data. Preliminary results that indicate density fluctuation levels and their spectral distribution will be presented.
\end{abstract}




\section{SECTION I}

\section{Background}

Interest in atmospheric turbulence dates to the first astronomical observations, which may be hampered by the random variations in index of retraction due to the turbulent changes in air density. More recently, flight through the atmosphere has generated significant interest in clear air turbulence as well as turbulence associated with meteorological phenomena and terrain variations. Desire to fly aircraft with laminar flow surfaces without atmospheric-turbulence-induced transition to turbulent flow has heightened this interest. The transport and dispersal of pollutants in the atmosphere are largely governed by near-surface turbulence. The production, convection and diffusion by $v$ iscous forces of atmospheric turbulence has long been of interest to basic researchers studying the atmosphere. With the advent of sophisticated detection and surveillance systems involving all wave lengths of radiation, the effect of atmospheric turbulence on the output of such systems has become important. The propogation of coherent radiation through the atmosphere can be seriously affected by the random phase shifts induced by atmospheric turbulence. Both the levels of the fluctuations and their size (or scale) are important in the effect that atmospheric turbulence will have on any of the above areas of interest. Investigations too numerous to cite have been conducted in the general study of atmospheric turbulence. Meteorological balloons are the classical system used for study. Variations in temperature, 
for example, are recorded with increasing altitude to yield the values of the fluctuating field. The scales of the fluctuations are determined typically by having two temperature sensors whose signals can be correlated which are separated by a known distance. Balloons give useful results and are usually quite reliable; however, they give information about the atmosphere over a very small volume. In addition, the scales that can be detected are limited to those larger than the separation distance between the sensors.

In other studies, fixed-wing aircraft have been used to increase the data base for atmospheric turbulence. A major program known as ALLCAT (all altitude critical air turbulence), supported by the Air Force, and other government agencies interested in atmospheric turbulence was undertaken around 1965. Data on gust loads sensed by gust probes, accelerometers, pressure transducers and other onboard instrumentation were taken. Aircraft ranging from the U-2 to F-106 to a $\mathrm{C}-131 \mathrm{~B}$ were used to investigate turbulence in various flight altitude bands. The LOCAT program investigated low altitudes -HICAT investigated high altitudes, etc. Results from these studies are presented in reports too numerous to give here, however, the interested reader is referred to Reference 1 and the bibliography given there. Because of the size of the aircraft used and relatively slow response instrumentation, only large scale motions could be accurately detected. Here large scale implies integral scale lengths greater than 10 to 20 meters. For interest in the statistics of atmospheric turbulence in the large scale region the results of the ALLCAT 
program represent an enormous data base obtained at a cost of millions of dollars. As extensive as these data may be, they do not contain useful information in the small turbulence scales, say from the order of 1 meter down to the dissipation scales of the order of millimeters.

To make accurate measurements of small-scale atmospheric motions from aircraft, high response instrumentation such as the hot-wire anemometer can be used. Previous experiments have been performed (see References 2, 3 and 4 for example) using hot-wire anemometry, however, the interpretation of the data resulting from these experiments is not clear because of the data reduction techniques employed. This is particularly true when attempting to measure the variations in atmospheric density and their scales for determining optical transmissibility. Since data for optical use is desired over extensive regions of the atmosphere at all flight altitudes, aircraft with large operating envelopes and high speeds must be used.

This report describes a system developed for atmospheric turbulence research by the USAF Weapons Laboratory at Kirtland AFB, New Mexico. The airborne platform for the system is an NC-135 aircraft used in various Air Force research projects. A pair of fine wire sensors are located near the nose of the aircraft (see Figures 1 and 2) and are driven with high response anemometer systems. Through the use of this aircraft, two independent sensors and data 
reduction procedures developed for high speed wind tunnel testing, both large and small scale turbulence (down to $5 \mathrm{~mm}$ ) can be measured at high speeds from near ground level to $12 \mathrm{~km}$.

\section{SECTION II}

\section{Discussion}

In order to accurately measure atmospheric turbulence down to scale sizes of the order of $10 \mathrm{~mm}$ in high speed flight, one requires instrumentation with a very high frequency response. For example, at a flight velocity of $200 \mathrm{~m} / \mathrm{s}$ a $10 \mathrm{kHz}$ response is needed to measure a turbulent burst $10 \mathrm{~mm}$ long. By comparison, if the aircraft slowed to $100 \mathrm{~m} / \mathrm{s}$, this same instrumentation could detect a $5 \mathrm{~mm}$ scale. Laboratory or wind tunnel instrumentation can easily operate today at up to 20 to $40 \mathrm{kHz}$. A system having this high response that can be used in making velocity and/or density turbulence measurements is the hot-wire anemometer. This system consists of a fine (typically less than $25 \mu \mathrm{m}$ diameter) wire, either heated or unheated, and the appropriate electronics (referred to as anemometers) to produce electrical signals that can be interpreted as fluctuations in the physical variables. A drawback of the hot-wire anemometer is the fragile nature of the sensors. Researchers have been reluctant to attempt using fine wires on aircraft because of this; however, techniques for producing durable sensors have been developed in high speed wind tunnel testing. Recently, a system using $5 \mu \mathrm{m}$ sensors and high response laboratory anemometers 
was used to survey the fuselage boundary layer of a large transport aircraft (Ref. 5). This system was used successfully in over 80 hours of flight time. Wire breakage occurred only once when ice crystals were encountered. With this encouraging record, it was decided to move the sensor location to the nose of the aircraft and attempt to make atmospheric turbulence measurements.

$$
\text { In making measurements at low speeds (i.e., } M<\cong 0.3 \text { ) }
$$

the signals produced by anemometer/sensor combinations are easily related to the fluid flow variables. For a constant density flow, the velocity fluctuations are sensed by a heated wire driven by either a constant temperature or constant current anemometer. Constant temperature systems are usually used at present because of their higher frequency response, although compensating amplifiers are available for use with the constant current systems. Fluid temperature (i.e., the aerodynamic static temperature) fluctuations are sensed by an unheated wire driven by a constant current anemometer, compensated to increase its frequency response.

As flight speeds increase above the $M \cong 0.3$ value, or the flow is no longer one of constant density, fluid compressibility effects become important and the values sensed by wires placed in the stream no longer respond to the simple velocity and fluid temperature. For example, consider the unheated or temperature sensing wire. Since the flow over the wire is essentially brought to rest at the wire surface, it will respond to the temperature known as the recovery 
temperature. This, in turn, can be related to the aerodynamic total temperature, which is related to the fluid static temperature through the energy relationship

$$
T_{t}=T_{t}+u^{2} / 2 c_{p}
$$

or

$$
T_{t}=T\left(1+\frac{\gamma-1}{2} M^{2}\right)
$$

Now it is evident that, for increasing Mach numbers, the difference between the total temperature (actually sensed by the wire) and the desired fluid temperature will increase. For a flight Mach number of 0.8 , there is about a $13 \%$ error in the mean value. Since, in atmospheric turbulence studies, we are interested in not the mean values but their indicated fluctuations, we must examine a differential form of equation (2).

$$
\left(1+\frac{\gamma-1}{2} M^{2}\right) \frac{T^{\prime}}{\bar{T}}=\frac{T_{t}^{\prime}}{\bar{T}_{t}}+(\gamma-1) M^{2} \frac{u}{\bar{u}}
$$

Equation 3 relates the instantaneous fluctuations away from the mean values (primed variables) than can exist at a point in the fluid. Again, for low Mach numbers we have

$$
\frac{T_{t}^{\prime}}{\bar{T}_{t}}=\frac{T^{\prime}}{\bar{T}}
$$

so that an unheated wire will sense the desired fluctuations in static temperature. For increasing Mach numbers, the values of the velocity fluctuations present in the atmosphere will contribute to the measured 
$T_{t}^{\prime}$ values. Keep in mind that the behavior described above comes about only through the aircraft's flight Mach number and has nothing to do with the motions and variations present in the atmosphere. If one were to use only a single, unheated wire, it would be impossible to measure the value of fluid temperature fluctuations from a high speed aircraft. Even to measure the spectrum of the fluctuations requires the assumption that the velocity and temperature spectra are identical. To surmount this apparent impasse, another wire, heated to at least twice the stream temperature, is required. This wire, when cooled by the air flow, becomes sensitive only to the mass flow past it. The mass flow is the product of fluid static density, $\rho$, and the velocity, $u$, past the wire. The term actually measured by the heated wire is ( $\left.\rho)^{\prime}\right) / \bar{u}$. For the small fluctuation levels usually encountered in turbulent flows, this term may be written as

$$
\frac{(\rho u)^{\prime}}{\bar{\rho} \bar{u}}=\frac{\rho^{\prime}}{\bar{\rho}}+\frac{u^{\prime}}{\bar{u}}
$$

Notice that the heated wire produces a signal proportional to the sum of two desired turbulence quantities $\rho^{\prime}$ and $u^{\prime}$. Once again, if one were to operate only a single, heated wire it would, in general, be impossible to determine the magnitude of the velocity fluctuations, since the density fluctuations from, say, temperature variations, would also produce a contribution to the signal. This is true independent of the aircraft's flight Mach number. Spectra can be obtained, however, under the assumption that the spectra of $\rho^{\prime}$ and $u^{\prime}$ are identical. 


\section{At this point we have the unique situation that at high} speeds we have two systems capable of measuring two fluid parameters, neither of which is desired to deduce information about the atmosphere, in general. In high speed wind tunnel testing, it has been known that combining the two measurements can yield the desired information about the fluctuating velocity and temperature or density. To do this we examine a form of Equation 3 that has used a differential form of the equation of state

$$
\frac{p^{\prime}}{\bar{p}}=\frac{\rho^{\prime}}{\bar{\rho}}+\frac{T^{\prime}}{\bar{T}}
$$

to give

$$
\left(1+\frac{\gamma-1}{2} M^{2}\right) \frac{T_{t}^{\prime}}{\bar{T}_{t}}=\frac{p^{\prime}}{\bar{p}}-\frac{\rho^{\prime}}{\bar{\rho}}+(\gamma-1) M^{2} \frac{u^{\prime}}{\bar{u}}
$$

Adding and subtracting $(\gamma-1) M^{2} \frac{\rho^{\prime}}{\rho}$ on the right-hand side of Equation (5) gives

$$
\left(1+\frac{\gamma-1}{2} M^{2}\right) \frac{T_{t}^{\prime}}{\bar{T}_{t}}=\frac{p^{\prime}}{\bar{p}}-\left(1+(\gamma-1) M^{2}\right)-\frac{p^{\prime}}{\bar{\rho}}+(\gamma-1) M^{2} \frac{(\rho u)^{\prime}}{\bar{\rho} \bar{u}}
$$

For small scale motions, the atmosphere is incapable of supporting pressure fluctuations (other than weak sound waves) so that the $\mathrm{p}^{\prime} / \overline{\mathrm{p}}$ term may be neglected in Equation 6. This leaves a relationship involving three fluctuation terms, two of which are measured: 


$$
\left(1+\frac{\gamma-1}{2} M^{2}\right) \frac{T_{t}^{\prime}}{\bar{T}_{t}}=-\left(1+(\gamma-1) M^{2}\right) \frac{\rho^{\prime}}{\bar{\rho}}+(\gamma-1) M^{2} \frac{(\rho u)}{\frac{\rho u}{-j}}
$$

so that the density fluctuation can be deduced. Phase lags between the two systems prevent the use of Equation 7 directly so that a timeaveraged equation must be used. We first write Equation 7 as

$$
A \frac{\rho^{\prime}}{\bar{\rho}}=B \frac{(\rho u)^{\prime}}{\bar{\rho} \bar{u}}-C \frac{T_{t}^{\prime}}{\bar{T}_{t}}
$$

and then square and time average to get

$$
A^{2} \frac{\overline{\rho^{\prime 2}}}{\bar{\rho}^{2}}=B^{2} \frac{\overline{(\rho u)^{\prime 2}}}{(\overline{\rho u})^{2}}-2 B C R_{\rho U T} \frac{\left\langle(\rho u)^{\prime}\right\rangle\left\langle T_{t}^{\prime}\right\rangle}{\overline{\rho u T} \bar{T}_{t}}+C^{2} \frac{\overline{T_{t}^{\prime 2}}}{\bar{T}_{t}{ }^{2}}
$$

Here $R_{\rho U T}$ is the zero-phase lag cross-correlation coefficient between ( $p u)^{\prime}$ ' and $T_{t}^{\prime}$, which is obtained from the recorded data. Thus $\rho^{\bar{T}} / \bar{\rho}^{2}$ can be determined.

Static temperature fluctuations instead of density fluctuations can be determined by using

$$
\overline{\frac{T^{12}}{\bar{T}^{2}}}=\overline{\frac{\rho^{12}}{\bar{\rho}^{2}}}
$$

Velocity fluctuations are obtained by using Equation 5 and adding and subtracting $u / / \bar{u}$ from the right-hand side to give 


$$
\left(1+\frac{\gamma-1}{2} M^{2}\right) \frac{T_{t}^{\prime}}{\bar{T}_{t}}=\frac{p^{\prime}}{\bar{p}}-\frac{(p u)^{\prime}}{\bar{\rho} \bar{u}}+\left(1+(\gamma-1) M^{2}\right) \frac{u^{\prime}}{\bar{u}}
$$

Again for small $\mathrm{p}^{\prime} / \overline{\mathrm{p}}$, we have

$$
A \frac{u^{\prime}}{\bar{u}}=\frac{(\rho u)^{\prime}}{\bar{\rho} \bar{u}}+c \frac{T_{t}^{\prime}}{\bar{T}_{t}}
$$

or

$A^{2} \frac{\overline{u^{\prime 2}}}{\overline{u^{2}}}=\frac{\overline{(\rho u)^{\prime 2}}}{(\overline{\rho u})^{2}}+2 C R_{\rho u T} \frac{\left.\left\langle(\rho u)_{t}\right)\right\rangle\left\langle T_{t}^{\prime}\right\rangle}{\overline{\rho u T} \bar{T}_{t}}+C^{2} \frac{\overline{T_{t}^{\prime}}}{\bar{T}_{t}{ }^{2}}$

Note that Equation (10) must be used to determine the magnitude of the velocity fluctuations even for very low-speed flight. These relationships were used in the present study to produce the results discussed next.

\section{SECTION III \\ Results}

Representative results from flights at various Mach numbers and altitudes are presented here and discussed in light of their implications for optical transmission through the measured atmospheric volumes. The data presented here were obtained on two flights made on 23 and 26 Feb, 1979. Conditions for the flights are given in Table 1. Data points are referenced throughout by file number. 


\begin{tabular}{|c|c|c|c|c|}
\hline File No. & Alt, $\mathrm{km}$ & $\overline{\mathrm{u}}, \mathrm{m} / \mathrm{s}$ & $\mathrm{M}$ & Date \\
\hline 1 & 3.66 & 234 & 0.57 & $2 / 23 / 79$ \\
2 & 3.66 & 153 & 0.48 & $2 / 23 / 79$ \\
3 & 3.66 & 124 & 0.37 & $2 / 23 / 79$ \\
4 & 3.66 & 93 & 0.28 & $2 / 23 / 79$ \\
5 & 8.84 & 271 & 0.83 & $2 / 26 / 79$ \\
6 & 11.89 & 237 & 0.80 & $2 / 26 / 79$ \\
\hline
\end{tabular}

Table I. Flight Conditions.

Expected optical degradation is desired from the present measurements of atmospheric turbulence. The random phase variations can be calcuated from the measured density fluctuations and integral length scale through a total distance by

$$
\sigma^{2}=2 \beta^{2} \overline{\rho^{12}}+L
$$

so that both the fluctuation levels and their scales must be known. The following discussion presents the method for determining them from the measured data.

A typical spectrum of the mass flux and total temperature (from File 1) in the frequency domain is shown in Figure 3. A -5/3 
slope is evident as low as $10 \mathrm{~Hz}$ and continues into the noise level at about $5 \mathrm{kHz}$. Since both the total temperature and mass flux fluctuations follow the Kolmogoroff energy cascade, then, through Equations 8 and 10, so will both the velocity and density fluctuations. For the flight speeds shown in Table 1; these frequencies correspond to scales from $10 \mathrm{~m}$ to $10 \mathrm{~mm}$. In optical applications, the integral scale of the density fluctuations are of interest and can be obtained easily by integrating the time auto-correlation function of either the mass flux or the total temperature to obtain the integral time scale and then using the mean velocity through the turbulence to calculate the integral spacial scale.

Spacial scales found for the present data are given below. The variation indicated for the $3.66 \mathrm{~km}$ data (Files 1-4) results from different values calculated as the flight speed changed by nearly a factor of 2.5. The relatively small variation gives one confidence that accurate scale size measurements can be obtained independent of flight speed. Presently used and conceived optical systems have apertures in the range of 0.5 to 2 meters, so that the relatively large turbulence scale to aperture ratio will produce low frequency rather than high frequency phase aberrations that may be compensated for by use of adaptive optical systems.

\begin{tabular}{|c|c|}
\hline Alt, km & I, integral scale, meters \\
\hline 3.66 & $6.0 \pm 5 \%$ \\
8.84 & 7.1 \\
11.89 & 10.9 \\
\hline
\end{tabular}


To determine the density fluctuation level, Equation 8 is used with measurements of mass flux and total temperature fluctuations along with their measured cross-correlation coefficient. For the data examined here, indicate that the cross-correlation between mass flux and total temperature fluctuations is essentially zero, so that the complexity of Equation 8 is reduced and the density fluctuations can be calculated:

$\left[1+(\gamma-1) M^{2}\right]^{2} \frac{\overline{\rho^{12}}}{\overline{\rho^{2}}}=\left[(\gamma-1) M^{2}\right]^{2} \frac{\overline{(\rho u))^{2}}}{\overline{\rho^{2} \bar{u}^{2}}}+\left[\left(1+\frac{\gamma-1}{2} M^{2}\right]^{2} \frac{\overline{T_{t}^{T^{2}}}}{\overline{T_{t}^{2}}}\right.$

Fluctuations in mass flux and total temperature were determined from the recorded data filtered at $5 \mathrm{kHz}$ to eliminate noise present in the signals beyond that limit. For the data analyzed here, the primary contribution to density fluctuations is from total temperature fluctuations. Even for the data, for which the Mach number was 0.83 , the error in neglecting the mass flux term was only $10 \%$. These findings are similar to those discussed by Levis (Ref. 6), although they may not hold universally. RMS data obtained in the present study are given below : 


\begin{tabular}{|c|c|c|c|}
\hline File No. & $\frac{\left\langle(\rho \mathrm{u})^{\prime}\right\rangle}{\bar{\rho} \bar{u}}, \%$ & $\frac{\left\langle_{\mathrm{T}}^{\prime}\right\rangle}{\overline{\mathrm{T}}}, \%$ & $\frac{\left\langle\rho^{\prime}\right\rangle}{\bar{\rho}}, \%$ \\
\hline 1 & 0.028 & 0.014 & 0.014 \\
2 & 0.026 & 0.011 & 0.011 \\
3 & 0.023 & 0.0067 & 0.0067 \\
4 & 0.025 & 0.0064 & 0.0064 \\
6 & 0.026 & 0.021 & 0.019 \\
\hline
\end{tabular}

With these data one can calculate the expected phase aberrations with Equation 11. The number of waves of distortion per kilometer are given in Table 2 for two wave lengths of interest.

\begin{tabular}{|c|c|c|}
\hline \multirow{2}{*}{ Altitude, } & \multicolumn{2}{|c|}{ Waves of Distortion } \\
\cline { 2 - 3 } & $\lambda=0.63 \mu \mathrm{m}$ & $\lambda=10.6 \mathrm{\mu m}$ \\
\hline 3.66 & $2.1-4.7$ & $0.12-0.28$ \\
8.84 & 3.9 & 0.23 \\
11.89 & 1.2 & 0.07 \\
\hline
\end{tabular}

Table 2. Expected optical distortion over $1 \mathrm{~km}$. 
The variation in atmospheric properties with altitude, location and time are evident in the wide range of values shown. Because of the large-scale of the turbulence, on target intensities for $10.6 \mu \mathrm{m}$ should be near diffraction limited even though $\lambda / 4$ aberrations are present. In calculating the velocity fluctuations from Equation 10 and the RMS values given above, it can be seen that up to a $100 \%$ error in $\left\langle u^{\prime}\right\rangle /_{\bar{u}}$ could result by neglecting the total temperature term in Equation 10.

\section{SECTION IV}

\section{Conclusions}

Atmospheric turbulence of scales down to $10 \mathrm{~mm}$ have been measured with an airborne system capable of making measurements up to altitudes of $12 \mathrm{~km}$. It is shown that both a heated and unheated wire are required in general to accurately determine either the velocity or density fluctuations. Further, for high speed flight, compressible effects on the instrumentation output must be considered to accurately deduce informaton about the atmosphere. Integral scales of the atmospheric turbulence can be accurately determined independent of flight speed. Typical data obtained to date show a $-5 / 3$ spectral decay to scales of 10 to $20 \mathrm{~mm}$. 


\section{References}

1. Loving, N.V., "Technical and Meteorological Planning to Meet the ALLCAT Program Objectives," in Clear Air Turbulence and Its Detection, edited by $Y-H$. Pao and $A$. Goldburg, Plenum Press, NY 1969, pp. 127, 143.

2. MacCready, P.B., Jr., "Turbulence Measurements by Sailplane," J. Geophysical Research, Vol 67, No. 3, March 1962.

3. Thompson, D.W., Coulter, R.L., and Warhaft, Z., "Simultaneous Measurements of Turbulence in the Lower Atmosphere Using Sodar and Aircraft," J. Applied Meteorology, Vol 17, June 1978, pp. 723, 734.

4. Lenschow, D.H., Friehe, C.A., and LaRue, J.C., "The Development of an Airborne Hot-Wire Anemometer System," Fourth Symposium on Meteorological Observations and Instrumentation, April 10-14, 1978, Denver, CO, published by the American Meteorological Society, Boston, MA, pp. 463-466.

5. Rose, W.C., Johnson, D.A. and Otten, L.J., III, "Application of Hot-Wire Anemometry and Laser Velocimetry to In-Flight Measurements of Turbulent Flow Properties," AlAA paper 78-825, 10th Aerodynmaic Testing Conference, San Diego, CA, April 19-21, 1978.

6. Levis, C.A. and Serafin, J.P., Analysis of Aero-Optical Turbulence Measurements, Final report for Contract F29601-78-C-0024, September 1978. 


\section{ABBREVIATIONS AND SYMBOLS}

\begin{tabular}{ll} 
L & path length through turbulence \\
\hline & correlation length \\
M & Mach number \\
p & pressure \\
$T$ & fluid temperature \\
U & velocity \\
$\beta$ & Gladstone-Dale constant \\
$\gamma$ & ratio of specific heats \\
$\lambda$ & wave length of radiation \\
$\rho$ & fluid density \\
$\sigma$ & optical phase variance
\end{tabular}

Superscripts

$\begin{array}{ll}() & \text { fluctuation in a quantity } \\ (-) & \text { time average of a quantity } \\ \langle()\rangle & \text { rms of a quantity }\end{array}$

Subscripts

t

total or stagnation conditions 


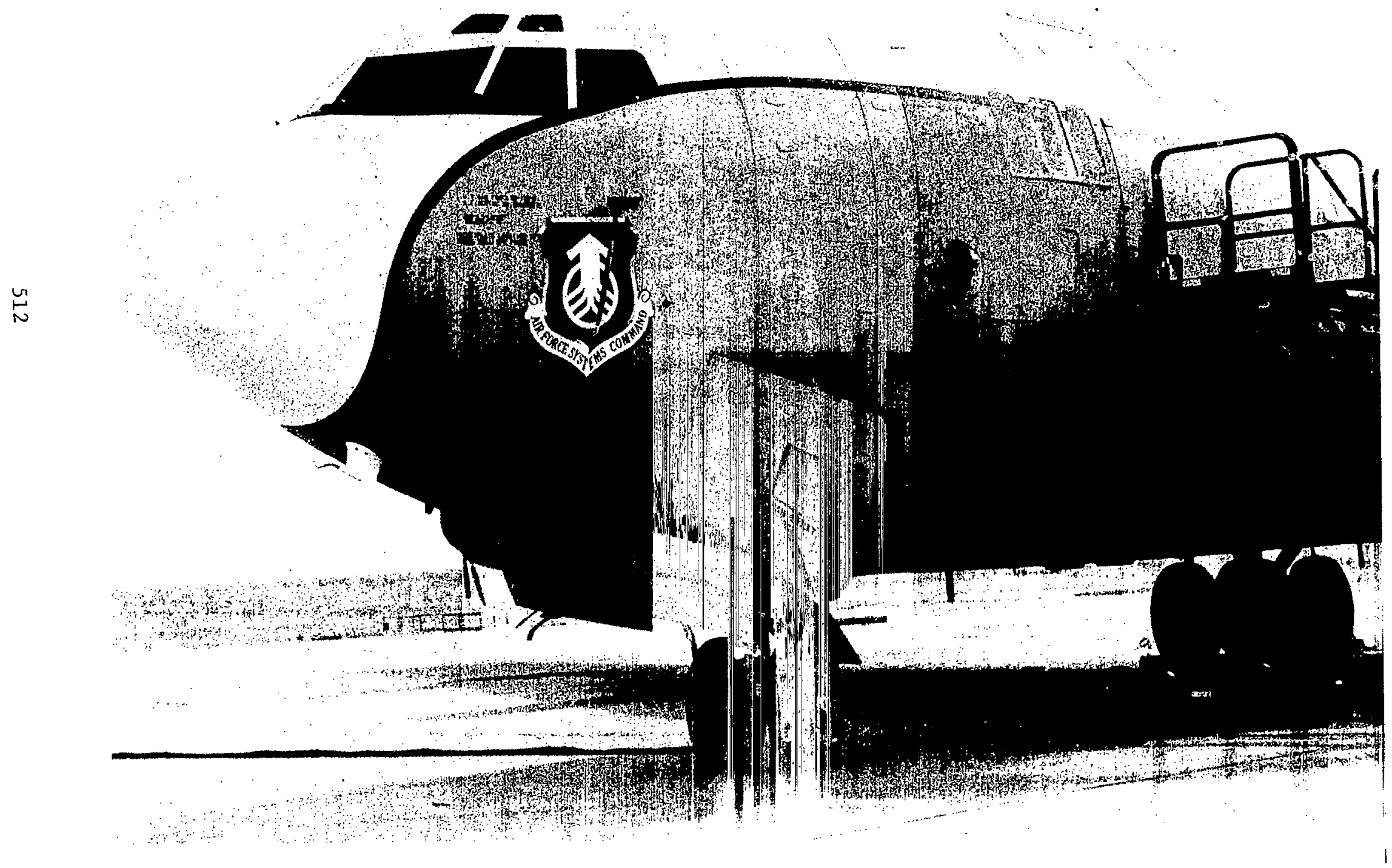

Figure 1. USAF Research Aircraft. 


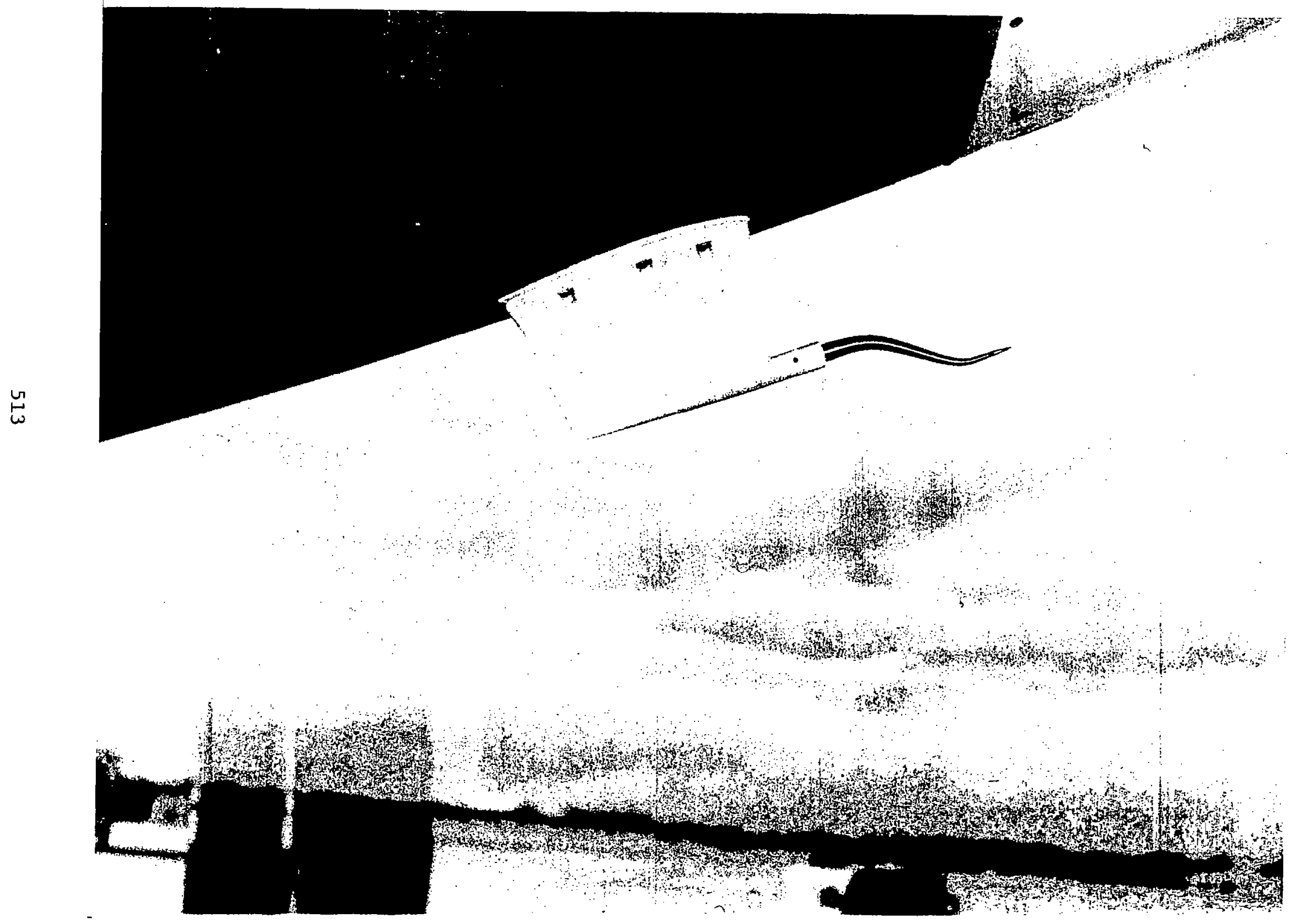

Figure 2. Closeup Showing Probe Installation and Sensor. 


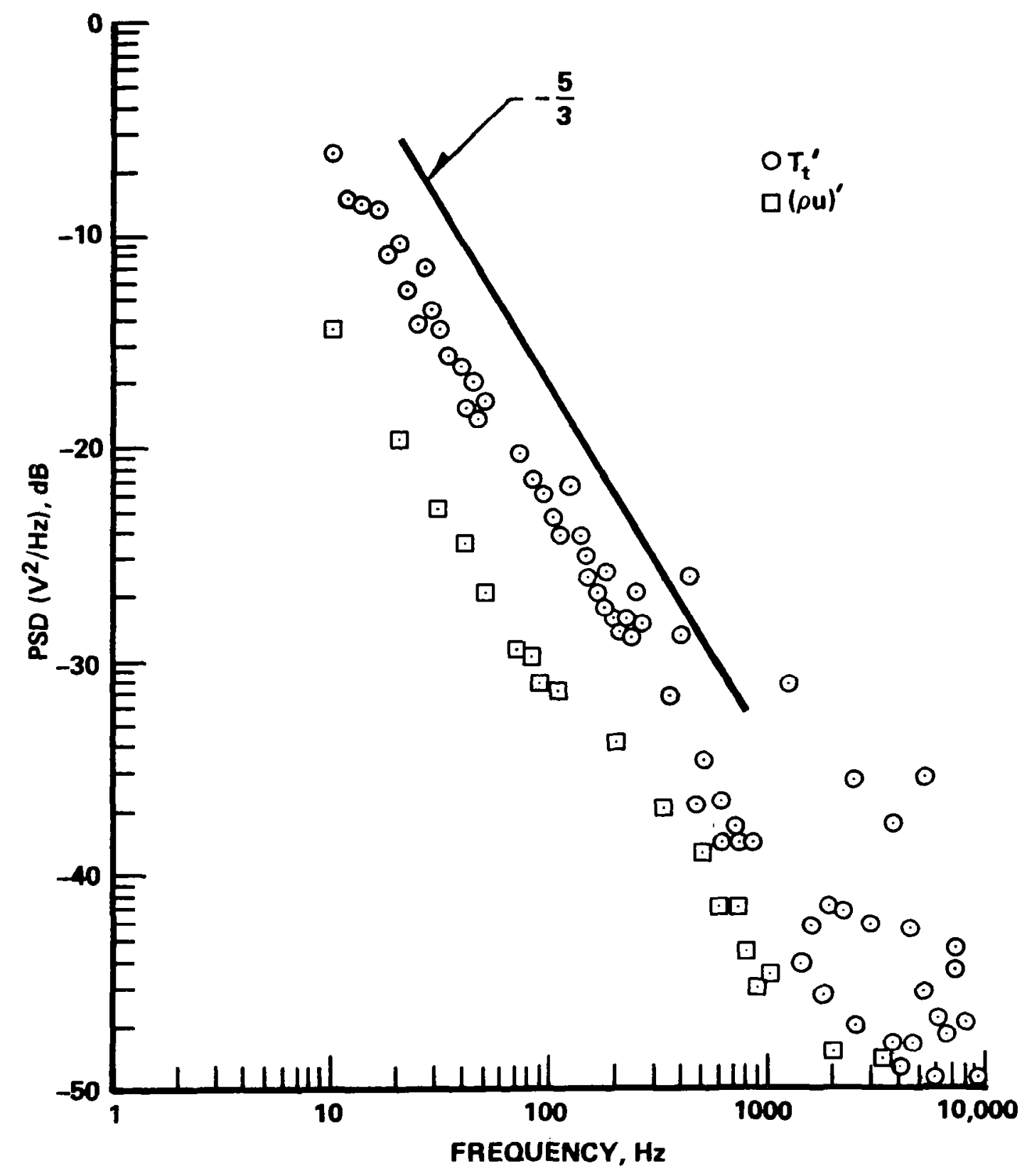

Figure 3. Spectra of Mass Flux and Total Temperature Fluctuations, $3.6 \mathrm{~km}, M=0.57$, 23 February 1979. 